\title{
LA NECESIDAD DE MANTENIMIENTO DE PERSONAS EN EMPRESAS DE LIMA METROPOLITANA
}

\author{
THE NEED FOR MAINTENANCE OF PEOPLE IN BUSINESS LIMA \\ Ampelio Ricardo Barrón Aráoz* \\ Docente Principal de la Facultad de Ciencias Contables \\ Universidad Nacional Mayor de San Marcos-UNMSM / Lima-Perú \\ [Recepción: Marzo de 2014/ Conformidad: Mayo 2014]
}

\section{RESUMEN}

El mantenimiento de personas consiste en la higiene, seguridad y calidad de vida laboral, temas que han sido abordados en el Proyecto de Investigación desarrollado el año 2013.

La higiene laboral está relacionada con las condiciones ambientales de trabajo que garantizan la salud física y mental; asimismo, con las condiciones de bienestar de las personas.

Desde el punto de vista de la salud física, el sitio del trabajo constituye el área de acción de la higiene laboral e implica aspectos ligados a la exposición del organismo humano a agentes externos, tales como: ruido, aire, temperatura, humedad, iluminación y equipo de trabajo.

Desde el punto de vista de la salud mental, el ambiente de trabajo debe establecer condiciones psicológicas y sociológicas saludables, que actúen de modo positivo sobre el comportamiento de las personas para evitar efectos emocionales como el estrés.

El término de calidad de vida laboral (CVL) fue acuñado por Louis Davis en la década de 1970, cuando desarrollaba un proyecto sobre diseño de cargos. Esta referencia lo hace Peter Drucker en su libro "Los Desafíos de la Administración en el siglo XXI”.

\section{Palabras clave:}

Higiene laboral; salud ocupacional; seguridad; calidad de vida laboral.

\begin{abstract}
Maintaining people is the health, safety and quality of work life issues that have been addressed in the research project 2013.

Occupational hygiene is related to environmental conditions which guarantee the physical and mental health, also with the welfare conditions of the people. From the point of view of physical health, the site of labor constitutes the action area of occupational hygiene and involves aspects related to the human body exposure to external agents such as noise, air, temperature, humidity, lighting and equipment working. From the point of view of mental health, the work environment must establish healthy psychological and sociological conditions that act positively on the behavior of individuals, to avoid effects such as emotional stress.

The term quality of work life (QWL) was coined by Louis Davis in the 1970s when developing a project on design fees. This reference does Peter Drucker in his book "Management Challenges in the XXI century".
\end{abstract}

\section{Keywords:}

Occupational hygiene; occupational health; security; quality of working life.

\footnotetext{
* Doctor en Ciencias Contables y Empresariales - UNMSM. Master en Administración de Empresas - UAM - España. Email: ricardo.20octubre@hotmail.com
} 


\section{INTRODUCCIÓN}

El presente artículo es el extracto del proyecto de investigación desarrollado el año 2013, cuyo título fue "La importancia de higiene, seguridad y calidad de vida en algunas empresas de Lima Metropolitana”.

La administración de personal en una organización, llámese privada o pública, comprende 6 momentos: Admisión, aplicación, compensación, desarrollo, mantenimiento y evaluación de personas.

En este artículo, nos ocuparemos solo del $5^{\circ}$ momento; es decir, el mantenimiento de personas, de la gestión del talento humano, empezando por la higiene laboral que trata sobre las condiciones ambientales de trabajo que garantizan la salud física y mental de las personas.

Como se ha señalado anteriormente, en lo que concierne a la salud física, el sitio de trabajo constituye el área de acción de higiene laboral e implica aspectos ligados a la exposición del organismo humano a agentes externos como: ruido, aire, temperatura, humedad, iluminación y equipo de trabajo.

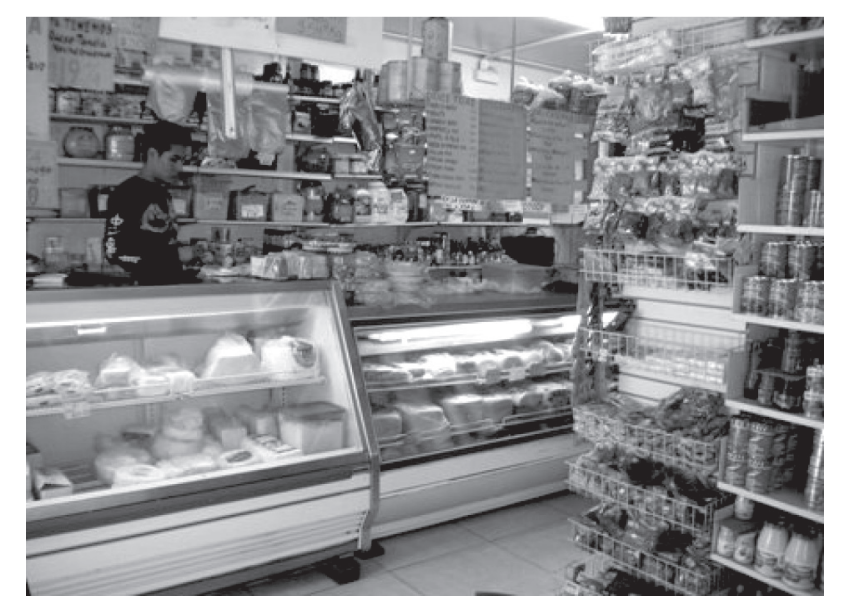

Sector donde se realizó el estudio.

En consecuencia, un ambiente saludable de trabajo debe brindar las condiciones ambientales físicas, que actúen de manera positiva sobre todos los órganos de los sentidos del hombre (vista, oído, olfato, tacto y gusto).

Respecto a la salud mental, el ambiente de trabajo debe establecer condiciones psicológicas y sociológicas saludables que actúen positivamente sobre el comportamiento de las personas, para evitar efectos emocionales, como el caso del estrés.
En la década de 1970, el término de calidad de vida laboral (CVL) fue acuñado por Louis Davis cuando desarrollaba un proyecto sobre el diseño de cargos. El concepto de calidad de vida laboral se refiere a la preocupación por el bienestar general y la salud de los trabajadores en desempeño de sus tareas.

Las empresas no solo deben pensar en atender bien al cliente externo; sino, no deben olvidar al cliente interno que son los trabajadores, ahora denominados colaboradores. El consultor danés Claus Moller sostiene que hoy en día, las empresas deben de poner a sus empleados en primer lugar, ya que por consecuencia, los mejores empleados pondrán a los clientes en primer lugar.

En el trabajo de campo, se ha empleado el método y las técnicas de entrevistas dirigidas a los dueños y gerentes de las empresas. No habiéndose aplicado encuestas por ser el tema muy especializado, ya que a cualquier persona no se le puede solicitar su opinión sobre el tema desarrollado.

De las entrevistas efectuadas y el material empleado en el marco teórico, se llegaron a conclusiones y recomendaciones importantes. Cabe señalar, que tuvimos serios problemas con las empresas grandes, debido a que no permitieron el ingreso a sus organizaciones para aplicar las entrevistas mediante un cuestionario diseñado a ese propósito.

Los objetivos fueron determinar qué porcentaje de empresas cumplen con el mantenimiento de las personas en el proceso de gestión de talento humano, consistente en la seguridad, higiene y calidad de vida.

Se justifica el trabajo, porque solo mediante una investigación científica se puedellegar a conclusiones, que la mayoría de los trabajadores están expuestos al deterioro de sus organismos e inclusive su vida, porque las empresas no cuentan con programas de prevención, para el cuidado de los aspectos físico y mental de sus colaboradores.

¿Por qué son importantes la seguridad y la salud de los empleados? Ofrecer un ambiente laboral seguro es importante por varias razones. Por ejemplo, lo sucedido en los EE.UU. de Norteamérica, donde recientemente se produjeron accidentes en el centro laboral y murieron 5,559 trabajadores. También, hubieron más de 3.8 millones de lesiones y enfermeda- 
des laborales que son resultados de los accidentes en el trabajo, aproximadamente 4.4 casos por cada 100 trabajadores anualmente.

Las estadísticas de México reflejan lo preocupante del descuido de las empresas. Durante el año 2002, la Secretaría del Trabajo y Previsión Social reportó que tuvieron 302,970 accidentes de trabajo en todo el país, lo cual representan $2.5 \%$ de accidentes laborales por cada 100 trabajadores. De esos accidentes, 13,384 resultaron con incapacidades totales y permanentes que representa $43.2 \%$ por cada 1,000 accidentes de trabajo.

Por otro lado, en lo concerniente a la salud ocupacional en ese mismo período se reportaron 4,511 enfermedades laborales, que como indicador representa $3.7 \%$ de tales enfermedades por cada 10,000 trabajadores. De estas enfermedades laborales 6,220 resultaron ser incapacidades permanentes. En total, 19,304 trabajadores dejaron de pertenecer a la población económicamente activa, por un descuido yla falta de prevención. En adición a esas cifras funestas, 1,049 personas murieron por accidentes de trabajo, lo cual representa $8.7 \%$ por cada 100,000 trabajadores.

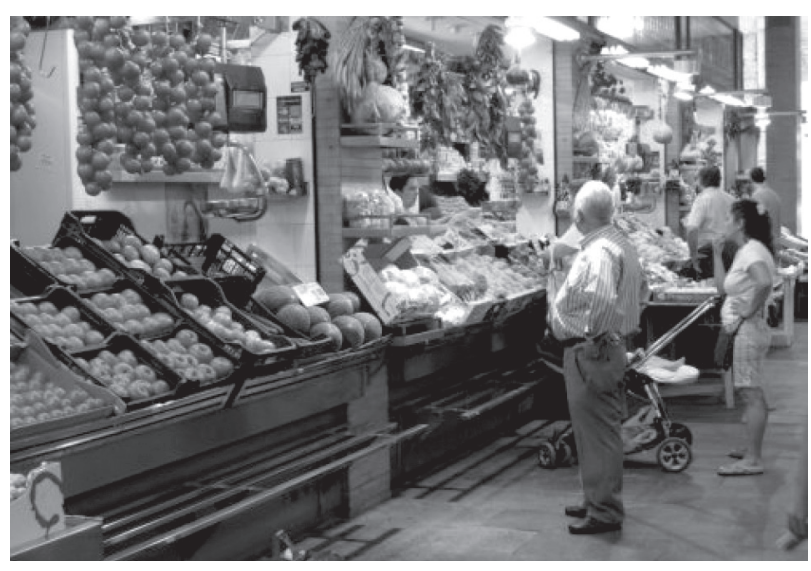

Mercado donde se realizó el estudio.

Es necesario tomar conocimiento de las siguientes definiciones:

\section{Salud ocupacional}

Una manera de definir la salud ocupacional es la ausencia de enfermedades. Sin embargo, riesgos de salud físicos y biológicos; tóxicos y químicos, así como, condiciones estresantes pueden provocar daños a las personas en el trabajo. El ambiente de trabajo en sí, también puede generar enfermedades.

\section{Higiene en el trabajo}

Se refiere al conjunto de normas y procedimientos que busca proteger la integridad física y mental del trabajador, preservándolo de los riesgos de salud inherentes a las áreas del cargo y al ambiente físico en que ejecuta las labores. Se relaciona con el diagnóstico, la prevención de las enfermedades ocupacionales, a partir del estudio y control de dos variables: el hombre y su ambiente de trabajo.

Los principales problemas de salud en las organizaciones están relacionados con:

1. Alcoholismo y dependencia química de drogas, medicamentos, tabaquismo, etc.

2. SIDA: Síndrome de inmunodeficiencia adquirida, que ataca el sistema protector del organismo contra enfermedades.

3. Estrés en el trabajo.

4. Exposición a productos químicos peligrosos, como ácidos, asbesto, etc.

5. Exposición a condiciones ambientales frías, cálidas, contaminadas, secas, húmedas, ruidosas, poco iluminadas, etc.

6. Hábitos alimentarios inadecuados: obesidad o pérdida de peso.

7. Vida sedentaria sin contactos sociales ni ejercicios físicos.

8. Automedicación sin cuidados médicos adecuados.

\section{Estrés en el trabajo}

El estrés es un conjunto de reacciones físicas, químicas y mentales de la persona frente a estímulos o elementos productores de tensión en el ambiente. Es una situación dinámica en que una persona enfrenta una oportunidad, restricción o demanda relacionada con lo que desea. El autoritarismo del jefe, la desconfianza, la presión de las exigencias y los cobros, el cumplimiento del horario de trabajo, el aburrimiento y la monotonía de ciertas tareas, la baja moral de los trabajadores, la falta de perspectiva del progreso profesional y la insatisfacción personal no solo reducen el buen humor de las personas, sino que también, provocan estrés en el trabajo. El estrés es la suma de las perturbaciones orgánicas y psíquicas provocadas por diversos agentes agresores. 


\section{¿Cómo reducir el estrés en el sitio de trabajo?}

Northwestern National Life Insurance Co. proporciona algunas guías:

1. Permita que los empleados conversen amigablemente entre sí.

2. Reduzca conflictos personales en el trabajo.

3. Dé a los empleados el control sobre cómo hacer su trabajo.

4. Hable abiertamente con los empleados.

5. Apoye los esfuerzos de los empleados.

6. Reduzca la cantidad de papeleo para los empleados.

7. Reconozca y recompense a los empleados.

\section{Seguridad en el trabajo}

Es el conjunto de medidas técnicas, educativas, médicas y psicológicas utilizadas para prevenir accidentes; eliminar las condiciones inseguras del ambiente, instruyendo o convenciendo a las personas sobre la necesidad de implantar prácticas preventivas.

La seguridad en el trabajo está relacionada con la prevención de accidentes y con la administración de riesgos ocupacionales. Su finalidad es profiláctica porque anticipa los riesgos de accidentes, para minimizarlos. En algunas organizaciones, la seguridad en el trabajo es prioridad fundamental.

Es el caso de DuPont, que comenzó como fabricante de explosivos en el curso del siglo XIX. En la fábrica de poliéster en Alemania, todas las mañanas el director general y los gerentes se reúnen a las 8:45 a.m. para revisar lo acontecido el día anterior. El principal tema de discusión no es la producción, sino la seguridad. Solo después de analizar los informes de accidentes y las medidas tomadas, los dirigentes comienzan analizar asuntos como calidad, producción y costos.

El índice de accidentes en DuPont es 0,12 accidentes por cada 100 empleados, frente a la media anual de 23 del National Safety Council, estimada para todos los fabricantes estadounidenses. Si DuPont estuviese en esa media general, gastaría más de US\$ 26 millones en costos de accidentes, lo cual representa 3,6\% de sus utilidades. Para cubrir esta diferencia, DuPont tendría que aumentar sus ventas en US\$ 500 millones y buscar un retorno líquido de $5,5 \%$.

\section{Calidad de Vida Laboral (CVL)}

En la actualidad, el concepto de CVL incluye tanto los aspectos físicos y ambientales como los aspectos psicológicos del sitio de trabajo. La CVL asimila dos posiciones antagónicas: por un lado, la reivindicación de los empleados por el bienestar y la satisfacción en el trabajo y, por el otro, el interés de las organizaciones por sus efectos potenciales en la productividad y la calidad de vida.

\section{TRABAJO DE CAMPO}

Se aplicó el método de entrevistas a los gerentes, dueños y funcionarios de las siguientes empresas: Fábrica de Escobas y Escobillas S.A.C. (Pequeña); Fábrica de Camisas Éxito EIRL (Mediana); Venta por menor de pollos del Mercado La Aurora (Pequeña); Venta de Abarrotes al por Mayor Comercial Perú Alto SRL (Mediana).

Ha sido muy difícil ingresar a la Gran empresa, al parecer no deseaban comprometerse con el vulnerable tema de seguridad, higiene y calidad de vida laboral. Igualmente, no se ha obtenido el universo de empresas (Población) de Lima Metropolitana, debido a que cada Municipio distrital tiene su propio registro de empresas. INEI, no tiene actualizado sus datos, la información que manejan corresponde al 2007, año del último censo.

Las entrevistas se realizaron en la segunda semana de noviembre de 2013. La muestra calculada ha sido de cuatro empresas, un promedio de 12 personas entre los dueños, gerentes y funcionarios. 
A continuación presentamos las entrevistas realizadas a los responsables de las entidades mencionadas:

Fábrica de Escobas y Escobillas S.A.C.

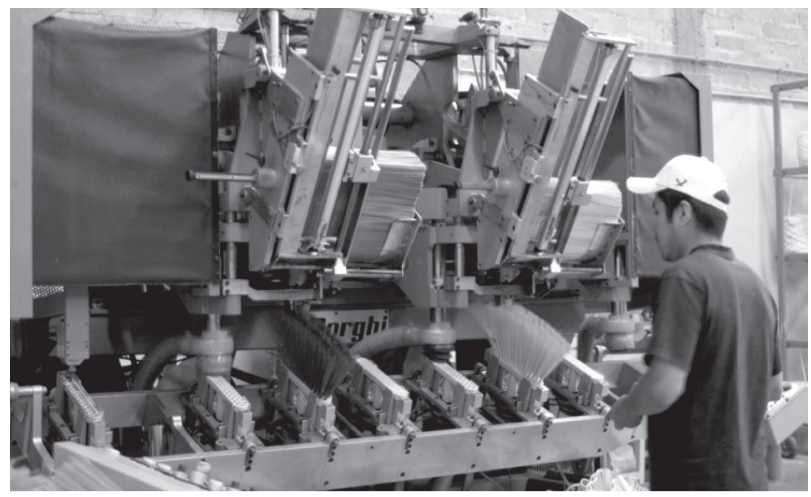

1. ¿La empresa que Ud. dirige, tiene previsto proteger la integridad física y psicológica de los trabajadores?

Respondieron: Que ellos protegen a los trabajadores sobre aspectos físicos y psicológicos, recurriendo a médicos especializados para que dicten charlas sobre el cuidado que deben tener los trabajadores tanto en salud física como el equilibrio emocional. Esto último estaría cubriendo la salud psicológica.

Los entrevistados no tienen ni idea de que la empresa por Ley, le debe brindar las condiciones ambientales y físicas adecuadas para proteger la vida de los trabajadores. Igualmente, desde el punto de vista de salud mental, también le debe brindar las condiciones psicológicas y sociológicas para que actúen de modo positivo sobre el comportamiento de personas para evitar efectos emocionales como es el caso del estrés.

\section{2. ¿Existe en su empresa el buen trato al personal} para evitar el estrés laboral?

Respondieron: Que gracias a las charlas de médicos especialistas dirigidos a los respectivos Jefes de Departamentos de la empresa, no se ha presentado situaciones de estrés de los trabajadores.

De la misma manera, las respuestas no cubrieron los objetivos planteados.

3. ¿Si se presentaran los accidentes, será por la irresponsabilidad de la empresa o por el descuido del propio trabajador?

Respondieron: Si se presentan esas situaciones no esperadas será por la negligencia del propio traba- jador. Muy indiferente la respuesta, en el sentido de aseverar que los trabajadores son responsables de los accidentes.

\section{4. ¿Si la empresa, debe cuidar que no se presenten deterioros físicos y psicológicos de los trabaja- dores?}

Respondieron: Que sí, porque los trabajadores son seres humanos al igual que nosotros, por ende siempre estamos pendiente de ellos. Hay solo buenas intenciones, pues no proveen los medios necesarios de protección.

\section{Fábrica de camisas ÉXITO EIRL}

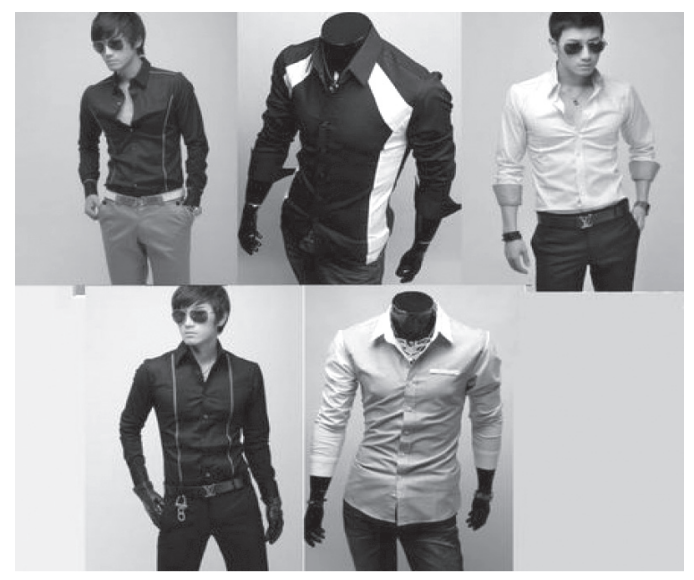

Se entrevistó al dueño del negocio y a 3 funcionarios.

\section{1. ¿̇e tiene previsto la integridad física y psicoló- gica de los trabajadores?.}

Respondieron coincidentemente: Somos responsables de los trabajadores, a quienes protegemos la salud física. La parte psicológica no nos interesa. La protección es a medias, no les importa la salud mental de los trabajadores de su empresa.

2. Sobre el estrés de los trabajadores, por el trato no adecuado al personal por parte de los Jefes o dueños del negocio. ¿ En su empresa se presenta esta situación?

Respondieron coincidentemente: En este negocio no provocamos el estrés del trabajador; si vemos al trabajador algunas veces malhumorado, creemos que esto obedece a causas extrañas al centro de trabajo.

$\mathrm{O}$ sea, que todo marcha muy bien en este negocio. 


\section{3. iSi se presentan accidentes en el trabajo es por la irresponsabilidad de la empresa o por descuido del propio trabajador?}

Respondieron: En la mayoría de los casos se debe a la negligencia del propio trabajador.

El trabajador es el que genera los accidentes, de este modo, se inhiben de su responsabilidad como empresa.

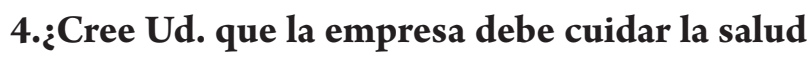
física y psicológica de sus trabajadores?

Respondieron: La empresa tiene responsabilidad de velar por el cuidado físico y psicológico de los trabajadores, para garantizar el buen desempeño.

Igualmente, la respuesta es genérica. Ellos responden por la protección de los trabajadores; pero no dicen de qué forma.

\section{Comercialización de pollos del mercado}

La Aurora

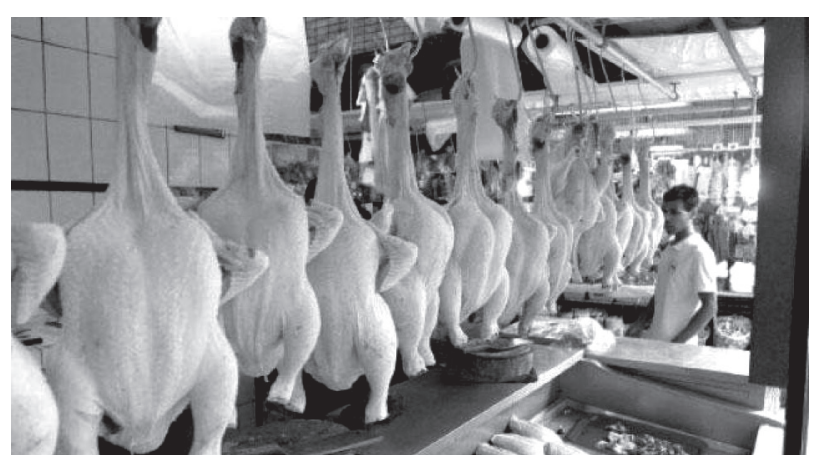

Se efectuó la entrevista a la vendedora de pollos del Mercado de La Aurora, con las siguientes preguntas:

\section{1. ¿Este negocio tiene previsto la integridad física y psicológica de sus trabajadores?}

Respondió la entrevistada: Tenemos cinco trabajadores a nuestro cargo. No conocemos normas del gobierno para el cuidado de salud física del personal; pero, sí se entrega reglas concretas para el cuidado de salud, sobre todo en el manejo de cuchillos filudos.

Estamos operando en el negocio más de ocho años, felizmente no se ha producido consecuencias fatales en cuanto a la salud física de los trabajadores; solo algunos accidentes pequeños, que han sido resueltos inmediatamente.
Desconocen las disposiciones sobre el cuidado del personal, pero a su manera dan reglas para el manejo de equipo (cuchillo).

2. En cuanto al estrés del trabajador. ¿Existe maltrato de parte de Ud. hacia los trabajadores para que cumplan sus funciones?

Respondió la entrevistada:

Tengo trabajadores jóvenes que cumplen sus funciones en forma adecuada. Mantenemos buenas relaciones, tanto a nivel de ellos mismos, así como, con la gerencia. No se han presentado casos de estrés, acá practicamos la solidaridad y compañerismo.

Tiene buen criterio para tratar al personal, justamente el maltrato conduce al estrés.

3. ¿Si se presentan o presentara los accidentes, sería por la irresponsabilidad de la empresa o por descuido de los propios trabajadores?

Respondió la entrevistada: Si se presentara esos momentos no deseables serían por la negligencia de los mismos trabajadores.

4. ¿Cree Ud. que la empresa debe cuidar la salud física y psicológica de los trabajadores?

Respondió la entrevistada: Nosotros sí nos preocupamos por la salud física de nuestros trabajadores, es por eso que no tenemos accidentes.

Hay coherencia, que sí se preocupan por la salud física de los trabajadores; pero la parte psicológica se ignora.

\section{Comercial PERU ALTO SRL}

La empresa se dedica a la venta de abarrotes al por mayor.

Se entrevistó al Administrador y a otro funcionario,

1.- :Tiene Ud. previsto proteger la integridad física y psicológica de sus trabajadores ?

Respondieron: En cuanto a la protección física, sí hemos habilitado a los trabajadores con uniformes, overoles y guantes, porque están expuestos a daños físicos con el cargado de sacos de mercaderías, en la distribución de productos a los clientes.

Como es una empresa comercial, proveen ciertos equipos para proteger la salud física del trabajador; 
pero igual que en los casos anteriores, no conocen las disposiciones vigentes para proteger la salud psicológica de los trabajadores.

\section{Sobre el estrés. ¿Existe en esta empresa el mal- trato de los Jefes o los dueños a los trabajadores?}

Respondieron: En esta empresa no existe tratos inhumanos, más bien acá practicamos la cordialidad y el respeto recíproco entre los trabajadores y los jefes o dueños.

Es importante la declaración de los entrevistados, que en esta empresa no hay trato inhumano. De esta manera, en parte estarían cubriendo la salud mental.

\section{3. ¿Si se presentan o presentaran los accidentes, sería por la irresponsabilidad de la empresa o por el descuido de los trabajadores?}

La respuesta de los entrevistados fue terminante: Como las operaciones no son de alto riesgo, casi no tenemos los accidentes.

Por la naturaleza del negocio (comercial) no hay mayor riesgo. Por lo tanto, no responde a la pregunta que si hay accidentes, no se ubica responsables ya que los hechos pueden ser motivado por el trabajador o no previstos por la empresa.

\section{4. ¿Cree Ud. que a los trabajadores se les debe cuidar del deterioro físico y psicológico dentro de la empresa?}

Respondieron: Somos conscientes que debemos cuidar a nuestros trabajadores de cualquier eventualidad que pueda presentarse para el deterioro de la salud física y psicológica de los empleados.

Están atentos al cuidado del personal, lo mismo no tienen nociones de prever el ambiente adecuado, de acuerdo a las normas para evitar los accidentes.

\section{CONCLUSIONES}

1. De acuerdo a las personas entrevistadas llámese propietarios o gerentes de las empresas, la mayoría de ellos, no tienen nociones ni idea de que existen normas legales que obligan enfáticamente el proceso de mantenimiento del talento humano en las empresas; es decir, seguridad, higiene laboral y calidad de vida laboral.
2. La mayoría de las personas entrevistadas manifestaron que sí tienen el debido cuidado para evitar que se produzcan accidentes, en deterioro de la salud física y mental de los trabajadores en el momento de trabajo; pero lo hacen a su manera, mediante charlas a cargo de médicos u otros especialistas en estos temas, mas no tienen previsto lo que ordena la Ley, como es el de adecuar los ambientes de trabajo para evitar el deterioro de la salud física y mental de los empleados.

3. En cuanto a la pregunta, si algunas veces se produjeron accidentes en su empresa, manifestaron tajantemente que no hubieron; si se produjeran estos no serían de responsabilidad de la empresa, sino del trabajador.

\section{RECOMENDACIONES}

1. Que el proceso de mantenimiento del talento humano es fundamental en los centros de trabajo. Como quiera que la mayoría de los negocios desconocen esta parte del proceso de gestión del personal, la entidad encargada, en este caso, el Ministerio de Trabajo debe ser más severo en supervisar el cumplimiento de las normas vigentes, o sea la higiene laboral, seguridad y calidad de vida laboral desarrollada dentro de las empresas.

2. En las universidades, en la currícula de estudios, se debe incluir una asignatura que comprenda sobre el mantenimiento de personal, en la Administración del talento humano sobre la seguridad, higiene laboral y calidad de vida humana laboral.

3. Que la Dirección o Departamento del Centro de Extensión Universitaria y Proyección Social - CEUPS de las universidades, debe organizar cursos dirigidos a los propietarios y gerentes de empresas de diversos tamaños, sobre la responsabilidad que tienen en la Administración del Talento Humano, el cumplimiento de las normas vigentes sobre la seguridad, higiene laboral y calidad de vida laboral. 


\section{REFERENCIAS BIBLIOGRÁFICAS}

1. CHIAVENATO, I. (2009). Gestión del Talento Humano. Ed. México Mc Graw.

2. DESSLER, G. y VARELA, R, (2011). Administración de los Recursos Humanos. México: Mc Graw-Hill.

3. DOMÍNGUEZ, H. (2006). El servicio Invisible. Bogotá, Colombia: Ecoe Ediciones. 